\title{
Active surveillance in prostate cancer management: where do we stand now?
}

\author{
Jędrzej Dragan, Jagoda Kania, Maciej Salagierski
}

Urology Department, Faculty of Medicine and Health Sciences,
University of Zielona Gora, Zielona Gora, Poland

Submitted: 22 December 2017; Accepted: 25 February 2018; Online publication: 3 March 2020

Arch Med Sci 2021; 17 (3): 805-811

DOI: https://doi.org/10.5114/aoms.2019.85252

Copyright $\odot 2019$ Termedia \& Banach

\section{Abstract}

Prostate cancer (PCa) is the most common cancer in men, with a steadily rising incidence, affecting on average one in six men during their lifetime. The increase in morbidity is related to the increasing overall life expectancy, prostate-specific antigen testing, implementation of new molecular markers for cancer detection and the more frequent application of multiparametric magnetic resonance imaging. There is growing evidence demonstrating that active surveillance (AS) is an alternative to immediate intervention in patients with very low- and low-risk prostate cancer. Ongoing reports from multiple studies have consistently demonstrated a very low rate of metastases and prostate cancer specific mortality in selected cohorts of patients. As a matter of fact, AS has been adopted by many institutions as a safe and effective management strategy. The aim of our review is to summarize the contemporary data on AS in patients affected with PCa with the intention to present the most clinically useful and pertinent AS protocols.

Key words: active surveillance, prostate cancer, molecular markers, multiparametric magnetic resonance imaging.

\section{Introduction}

Prostate cancer ( $\mathrm{PCa}$ ) is the most frequently diagnosed non-skin cancer among European men [1]. This malignancy affects mainly senior men aged 60 and above. Factors known to be associated with the development and progression of PCa are age, family history, and ethnicity, with age being the most important one. Golabek et al. [2] also found that excess calcium intake (dietary supplements and food) may increase the risk level of PCa. Most of the diagnosed tumors are of indolent nature and, importantly, only a small number of prostatic malignancies have aggressive clinical behavior that requires immediate treatment.

In addition to the group of diagnosed patients, there is a large number of undiagnosed patients who do not experience any side effects of their illness. This is revealed in the results of post mortem examinations. In the group of men aged over 60 years, PCa was observed in more than $40 \%$ of cases. In the group of men over 80 years nearly $60 \%$ were harboring the disease $[1,3]$.

The standard treatment of PCa in intermediate- and high-risk groups remains radical prostatectomy or radiotherapy. Radical treatment is re-

\author{
Corresponding author: \\ Prof. Maciej Salagierski MD, PhD \\ Faculty of Medicine \\ and Health Sciences \\ University of Zielona Gora \\ Zyty 28 \\ 65-046 Zielona Gora, Poland \\ Phone: +48 6832831 05, \\ +48504174253 \\ E-mail: m.salagierski@wlnz. \\ uz.zgora.pl; \\ www.wlnz.uz.zgora.pl
}


lated to complications, which frequently significantly affects the patients' quality of life.

Active surveillance (AS) is a new strategy for the management of patients with low-risk PCa, aiming at postponing treatment until the cancer becomes clinically significant. The intention is both to increase patient quality of life by avoiding the consequences of treatment-related side effects and to lower socio-economic costs. This approach is based on the fact that most PCa patients are asymptomatic for life and treatments involve risks of major complications including erectile dysfunction and incontinence. Treatment of PCa is only implemented when the disease progresses.

Many diagnostic tests are used to assess the stage of disease, such as prostate biopsies, serum markers (PSA), magnetic resonance imaging (MRI) and genetic markers that are just being implemented and whose usefulness is only now being studied [4-7].

This review aims to gather knowledge about AS and to assess its practical clinical use along with the assessment of new indicators that could improve AS protocols [8].

\section{Evidence acquisition}

The authors of this review have systematically reviewed the PubMed database. From this database the 44 most recent articles on AS of PCa were selected, including incorporation of the latest National Comprehensive Cancer Network (NCCN) and European Urology (EAU) guidelines. Selection of articles was performed according to preferred reporting items for systematic reviews and meta-analysis statement guidelines. The review was about a comprehensive analysis of AS including methods that could help in its improvement. The authors searched for articles using the key words active surveillance, prostate cancer, molecular markers, mpMRI. The authors focused on selecting the latest publications (last 2 years). Among the previously published articles, those with a large number of citations were selected (min. 70).

\section{Active surveillance idea}

Active surveillance is a management strategy that aims to avoid invasive therapy among patients with clinically insignificant tumors [4, 7]. Instead of immediate treatment, regular diagnostics and follow-up investigations are started to assess the progression of the disease. The advantage of AS is to delay the side effects of treatment or to avoid them completely if the cancer does not become clinically significant. In addition, the patient, when postponing the treatment, can rely on the fact that when therapy is implemented in the future, new, potentially more effective, therapeutic options will be available [6].
It is of utmost importance to distinguish between AS and watchful waiting (WW). Both strategies involve postponing treatment, but there are significant differences between them. Active monitoring is intended for younger men - with a long, over 10 years life expectancy. In contrast, watchful waiting is for elderly men - with an estimated survival below 10 years. In addition, WW, unlike active monitoring, focuses on managing diseases that pose a direct threat to life and/or quality of life. Patients who decide to be vigilant may from time to time perform a PSA blood test, but there is no need to repeat the prostate biopsy. In the case of AS, the main focus is on the control of cancer progression and in this case invasive tests are not abandoned [8-10].

\section{Limitations of active surveillance}

The potential AS drawbacks involve: (1) a potential delay of the treatment of aggressive cancer (2) and psychological strain.

1) AS is characterized by a statistically higher incidence of disease progression and metastases than radical surgery or radiotherapy. However, according to the study results, at a median 10 years follow-up, active monitoring does not lead to an increase in PCa specific mortality [11, 12].

2) An inseparable aspect that should accompany AS should be care for the patient's psychological comfort. From the moment of diagnosis, the patient's life is often subordinated to medical procedures and the endless diagnostic process. In addition, the patient must struggle with uncertainty about his own future - never knowing when the disease will escalate. Patients with fear may refuse AS because they do not allow themselves to think about not treating the disease. The additional stress related to AS might decrease the comfort of life [13-15].

\section{Active surveillance protocols}

There are many protocols devoted to AS in the management of PCa. Only asymptomatic patients with clinically insignificant, low-volume neoplasms - characterized by slow growth kinetics - can be included in the AS protocol. The PCa division system uses the following criteria [7]:

- PSA blood concentration,

- prostate biopsy result based on the Gleason scoring system,

- T, N, M classification.

The risk group is determined based on the values of different parameters. Knowing the stage helps to decide what type of treatment would be the most appropriate for the patient and can help to predict the disease prognosis. Depending on the protocol (Table I [10, 11, 16-23]), different 
Table I. Available protocols for active surveillance programs

\begin{tabular}{|c|c|c|c|c|c|c|c|}
\hline \multirow[t]{2}{*}{ Study } & \multicolumn{4}{|c|}{ Monitoring protocols for active surveillance } & \multicolumn{3}{|c|}{ Criteria for active surveillance } \\
\hline & $\begin{array}{l}\text { Confirmatory } \\
\text { biopsy, from } \\
\text { diagnostic biopsy } \\
\text { [years] }\end{array}$ & $\begin{array}{l}\text { Repeat } \\
\text { biopsy } \\
\text { [years] }\end{array}$ & $\begin{array}{c}\text { PSA } \\
\text { testing } \\
\text { [month] }\end{array}$ & $\begin{array}{c}\text { Rectal } \\
\text { examination } \\
\text { [month] }\end{array}$ & $\begin{array}{l}\text { Clinical } \\
\text { stage }\end{array}$ & $\begin{array}{c}\text { PSA } \\
{[\mathrm{ng} / \mathrm{ml}]}\end{array}$ & $\begin{array}{l}\text { Gleason } \\
\text { score }\end{array}$ \\
\hline NCCN guidelines [10] & Undefined & $\begin{array}{l}\text { Not more } \\
\text { often than } 1\end{array}$ & $\begin{array}{l}\text { Not more } \\
\text { often } \\
\text { than } 6\end{array}$ & $\begin{array}{c}\text { Not more } \\
\text { often than } 12\end{array}$ & $\leq \mathrm{T} 2 \mathrm{~A}$ & $<10$ & $\begin{array}{c}\leq 6 \text { or } \\
\text { Gleason } \\
\text { grade group } 1\end{array}$ \\
\hline EAU guidelines [11] & Undefined & Undefined & Undefined & Undefined & cT1-2a & $<10$ & $<7$ \\
\hline $\begin{array}{l}\text { Bokhorst et al. } \\
\text { [16] (PRIAS, } \\
\text { multinational) }\end{array}$ & 1 & $\begin{array}{l}3 \text { for the } \\
\text { first } \\
10 \text { years }\end{array}$ & $3-6$ & - & $\leq \mathrm{T} 2$ & $\leq 10$ & $\leq 6$ \\
\hline $\begin{array}{l}\text { Welty et al. (UCSF, } \\
\text { San Francisco, CA) } \\
{[17]}\end{array}$ & 1 & $1-2$ & 3 & 6 & $\leq \mathrm{T} 2$ & $\leq 10$ & $\leq 6$ \\
\hline $\begin{array}{l}\text { Selvadurai et al. } \\
\text { (Royal Marsden } \\
\text { Hospital, London, } \\
\text { UK) [18] }\end{array}$ & $1.5-2$ & 2 & $3-6$ & $3-6$ & $\leq \mathrm{T} 2$ & $<15$ & $\begin{array}{c}\leq 6 \\
\text { or } 3+4=7\end{array}$ \\
\hline $\begin{array}{l}\text { Thompson et al. } \\
\text { (St. Vincent's } \\
\text { Prostate Cancer } \\
\text { Centre, Sydney, } \\
\text { Australia) [19] }\end{array}$ & 1 & $1-2$ & $3-6$ & $6-12$ & $\leq \mathrm{T} 2 \mathrm{a}$ & $<10$ & $\leq 6$ \\
\hline $\begin{array}{l}\text { Thomsen et al. } \\
\text { (University of } \\
\text { Copenhagen) [20] }\end{array}$ & 1 & Variable & 3 & 3 & $\leq \mathrm{T} 2 \mathrm{a}$ & $\leq 10$ & $\leq 6$ \\
\hline $\begin{array}{l}\text { Soloway et al. } \\
\text { (University of Miami, } \\
\text { Miami, FL) [21] }\end{array}$ & $<1$ & 1 & $3-6$ & $3-6$ & $\leq \mathrm{T} 2$ & $\leq 10$ & $\leq 6$ \\
\hline $\begin{array}{l}\text { Klotz et al. } \\
\text { (Sunnybrook Health } \\
\text { Sciences Centre, } \\
\text { Toronto, Canada) [22] }\end{array}$ & 1 & $3-4$ & $3-6$ & - & - & $\leq 10$ & $\leq 6$ \\
\hline $\begin{array}{l}\text { Tosoian et al. (Johns } \\
\text { Hopkins University, } \\
\text { Baltimore, MD) [23] }\end{array}$ & 1 & $1-2$ & 6 & 6 & T1c & - & $\leq 6$ \\
\hline
\end{tabular}

groups are distinguished, i.e. five risk groups according to the NCCN [10] and three as per EAU guidelines [11]. Despite the existence of various protocols, the classification groups for AS are characterized by the following criteria: PSA $<10$ $\mathrm{ng} / \mathrm{ml}, \mathrm{GS}<7$ and $\mathrm{cT} 1-2 \mathrm{a}$. The above-mentioned methods are used not only to include patients in the AS, but they are also used to assess the extent of the disease. The biopsy result is considered the most relevant, but it is far from ideal. Prostate biopsy is an invasive procedure, which might lead to complications, and it is not $100 \%$ accurate. That is why additional tools are being developed to improve the AS inclusion criteria and AS follow-up protocols. Monitoring in the AS consist of regular outpatient appointments, which usually involve digital rectal examination (DRE), PSA testing and biopsy. The frequency of these tests varies depending on the protocol.

\section{Diagnostic tools}

Every patient eligible for AS requires a precise diagnostic process and regular testing. The main aim of these actions is to assess the disease progression. Fundamental techniques used in diagnosing and monitoring patients with PCa are PSA, biopsy and multiparametric magnetic resonance imaging (mpMRI). Additionally, to increase the effectiveness of AS, there is a possibility to use genetic testing: PCA3 score, PHI score, 4K score, SelectMDx, ConfirmMDx and TMPRSS2-ERG gene fusion. Although the new genetic markers are not used in the current AS protocols, they appear very 
helpful in identifying men with potentially aggressive disease among patients with low PSA levels. Importantly, in the above group, biomarkers are able to correct for too low accuracy of PSA alone.

\section{Multiparametric magnetic resonance imaging}

The use of multiparametric MRI (mpMRI) allows early detection of clinically significant PCa. It combines T2-weighted imaging (T2WI), dynamic contrast-enhanced imaging (DCEI) and diffusion-weighted imaging (DWI), but T1-weighted imaging (T1WI) is additionally used to show any hemorrhage or other changes. The radiologist grades the lesion by the PI-RADS five-point scale. The lower the value, the less aggressive the lesion is:

- PI-RADS 1: very low (presence of clinically significant tumor is low probable),

- PI-RADS 2: low (presence of clinically significant tumor is low probable),

- PI-RADS 3: intermediate (presence of clinically significant tumor is ambiguous),

- PI-RADS 4: high (presence of clinically significant tumor is probable),

- PI-RADS 5: very high (presence of clinically significant tumor is probable) $[24,25]$.

These days, the second version of this algorithm has been developed and new studies about its utility are being performed. Hoffmann et al. [25] compared PI-RADSv1 and PI-RADSv2 and they did not find any difference in accuracy to predict clinically significant PCa. They also found that $\mathrm{mpMRI}$ accuracy in detecting anterior tumors was about $86-88 \%$. On the other hand, Kasel-Seibert et al. [26] found that when it comes to suspicious intraprostatic lesions, PI-RADSv2 could be a reliable tool, especially when its value is $\geq 3$. The Kasel-Seibert et al. study included experienced and inexperienced readers. The main aim of creating PI-RADSv2 was to improve diagnostic quality, and the study [27] confirmed that PI-RADSv2 was more accurate than PI-RADSv1. According to PI-RADSv2, a score $\geq 3$ appears to be much more specific, when considering if the patient should undergo repeated biopsy, than PSA level. The algorithm can also fail when it comes to anterior lesions - a malignant lesion can be described as benign by the radiologist following the algorithm, but the targeted biopsy appears positive, showing aggressive lesion. It can occur as a result of insufficient practice by the radiologists, as it is a relatively new method. According to many studies, PI-RADSV2 has a very high negative predictive value (NPV) and by itself remains an essential diagnostic tool. MpMRI may successfully confirm low-risk PCa, allowing repeat prostate biopsy to be avoided. The sensitivity of mpMRI in PCa diagnosis was $72.9-97.6 \%$ [28], the specificity $81.25 \%$ [29].

\section{Prostate biopsy}

Biopsy remains essential in PCa diagnosis. There are a several kinds of biopsy - standard, saturation and fusion biopsy. Referral to standard biopsy is based on elevated PSA levels and/or suspicious digital rectal examination (DRE). In a standard biopsy protocol, 12 samples are taken. Saturation biopsy is much more extended - it includes 20 or more samples and is performed under general anesthesia. Fusion biopsy is a relatively novel approach, which combines mpMRI and transrectal ultrasound (TRUS) images. A radiologist analyzes the mpMRI image and marks the boundaries of the lesion. While performing a biopsy, TRUS-MRI fusion imaging is used to take samples of the lesion, which was observed on MRI. Interestingly, Pepe et al. [30] compared saturation and fusion biopsy, as tools for monitoring patients during AS. The study revealed higher diagnostic accuracy of saturation biopsy than that of the fusion procedure. Although the detection rate was really high for saturation biopsy, which shows its utility, the lower detection rate for fusion biopsy might be due to the low quantity of samples (on average, 4 cores). Another study of Pepe et al. [31] included 75 men eligible for AS, who underwent mpMRI before confirmatory extended biopsy. Confirmatory biopsy is a follow-up test, to provide the most accurate diagnosis to the patient, and make sure there is no occurrence of underdiagnosing or underclassifying the lesion. The results showed that $28 \%$ of men were reclassified by saturation biopsy (30 cores) combined with fusion biopsy (4 cores), which proved Gleason score $\geq 7$. The authors stressed the importance of the combination of mpMRI and confirmatory biopsy, to obtain the most reliable diagnostic result.

\section{Markers}

\section{Prostate-specific antigen}

Prostate-specific antigen concentration although still frequently used remains a controversial clinical test to assess the risk of diagnosis of PCa. First of all, PSA lacks specificity. Secondly, it frequently leads to overdiagnosis and overtreatment of clinically insignificant disease. The current approach suggests that increased PSA levels should be combined with other diagnostic tools. Bancroft et al. [32] performed a study where men aged 40-69 years had PSA testing, and those with a PSA level > $3 \mathrm{ng} / \mathrm{ml}$ were offered a prostate biopsy. 162 men underwent a biopsy, 59 cancers (intermediate or high-risk) were detected and 42 of them were carriers of BRCA1 or BRCA2 gene mutation. That suggests a correlation between elevated PSA levels and a possible aggressive lesion in the prostate in BRCA1/2 mutation carriers.

The PSA test is not confined to total PSA plasma concentration - also pro-PSA level, PSA density and free PSA can be measured [10]. When it comes 
Table II. Diagnostic accuracy of PCa molecular markers

\begin{tabular}{|c|c|c|c|}
\hline Diagnostic tool & Sensitivity (\%) & Specificity (\%) & Author \\
\hline PCA3 & $47-66$ & $60-76$ & Nickens et al. [37] \\
\hline PHI score & $90-97.90$ & $31.1-38.00$ & Kretschmer et al. [38], Tosoian et al. [39], Loeb S et al. [40] \\
\hline SelectMDx: & & & Van Neste et al. [41] \\
\hline HOXC6 & 90 & 33 & \\
\hline DLX1 & 83 & 16 & \\
\hline HOXC6 + DLX1 & 91 & 36 & \\
\hline ConfirmMDx & 68.00 & 64.00 & Stewart et al. [42] \\
\hline
\end{tabular}

to aggressive tumors, elevation of pro-PSA levels seems to be a more accurate diagnostic tool [33]. PSA density is the quotient of total PSA level divided by prostate gland volume, and the higher it is, the higher is the likelihood of detecting a clinically significant disease [33].

\section{Prostate cancer antigen 3}

Prostate cancer antigen 3 (PCA3) is a non-invasive diagnostic tool describing expression of PCA3 RNA. During DRE the prostate massage is performed and the urine sample is taken after. This test is based on RNA PCA3/RNA ratio $\times 1000$. An elevated (higher than 35) PCA3 score correlates with a higher risk of positive prostate biopsy [34, 35]. PCA3 provides higher diagnostic accuracy than serum PSA concentration. Although the PCA3 test appears helpful in identifying men with potentially aggressive disease requiring immediate treatment, the prognostic value of the test has been questioned [36] (Table II [37-42]).

\section{Prostate health index score}

The prostate health index (PHI) score is a combination of three PSA isoforms: total PSA, ratio of total and free PSA (\%fPSA) and proPSA. It was created to improve the specificity of PSA testing [39]. However, $\mathrm{PHI}$ density, compared to the $\mathrm{PHI}$ score alone, is even more precise, showing $97.9 \%$ sensitivity and $38 \%$ specificity for clinically significant PCa $[38,39]$.

\section{Kscore}

The 4Kscore includes total plasma PSA, free PSA, intact PSA (inactive PSA form) and human kallikrein-2 (hk2). Kallikrein-2 is a protease whose expression was found in the prostate gland. Combining the 4Kscore with patient's age, DRE and biopsy results forms the 4 Kscore Test. This test is mainly used for assessing the risk level for diagnosing PCa $[9,38]$.

\section{SelectMDx}

The test measures the mRNA levels of the (urinary homeobox C6-HOXC6 and distal-less homeobox 1-DLX1) biomarkers, using kallikrein KLK3 expression as an internal reference, to aid in patient selection for prostate biopsy. Higher expression levels of DLX1 and HOXC6 mRNA are associated with an increased probability for highgrade Gleason score PCa. The assay is performed on post-DRE urine samples. Several recent studies revealed that using SelectMDx might reduce the overdiagnosis of clinically insignificant disease and by such means might lower the overtreatment of PCa [38].

\section{ConfirmMDx}

ConfirmMDx is an assay which checks the epigenetic alterations (precisely the methylation status) of many oncogenes (incl. Ras) from the patient's last negative biopsy. The basis of using ConfirmMDx is to discover the "halo effect" that tumor cells effuse by their epigenome, because it can be the very first symptom of a growing lesion that can be seen by the pathologist. ConfirmMDx may also be a predictor of a further negative biopsy $[9,38]$.

\section{TMPRSS2-ERG}

TMPRSS2-ERG is a fusion of TMPRSS2 and ERG genes, which can be detected in more than $50 \%$ of PCa patients. TMPRSS2 is a gene which encodes a serine protease. The exact function of this gene's protein is still unknown, but it is regulated by androgens. ERG is a transcription factor from the family of ETS factors, which controls cell differentiation and apoptosis. TMPRSS2-ERG gene fusion occurrence combined with elevated PCA3 improves both sensitivity and specificity of these tests in PCa detection [43]. Furthermore, TMPRSS2-ERG fusion may be associated with resistance to androgen deprivation therapy [44].

\section{Cost-effectiveness}

To assess the cost-effectiveness of AS, many factors have to be taken into account. In the case of patients without progression of the disease, no costs associated with the therapy will arise at all. Howev- 
er, one should take into account the costs resulting from the necessity to carry out regular diagnostic tests. It should be mentioned, however, that such tests would also be performed in people after the therapy as recurrence control instead of prognosis control. The potential additional cost of AS seems to be limited only to a group of patients who are not properly diagnosed and whose treatment costs will probably be higher due to the late start of treatment. The health aspect should also be taken into account when continuing the AS assessment. Due to postponing the treatment, the life of patients will not worsen as a result of its side effects. As a consequence, they will maintain full efficiency of work and will not require financial outlays in the form of allowances and maintenance bonuses.

\section{Conclusions}

Active surveillance seems to be an appropriate solution for selected patients harboring a low risk PCa. Compared to radical treatment AS: (1) helps to avoid unnecessary treatment and (2) enables maintaining the quality of life together with normal activities. In addition, (3) AS allows the costs of treatment to be reduced. The potential drawbacks of AS which should be considered include: (1) psychological strain and (2) a potential delay of the treatment of aggressive cancer. There is also (3) a need for regular follow-up investigations including frequent imaging studies and biopsies. The authors of the review recommend the use of AS for every patient diagnosed with low-risk prostate cancer meeting the inclusion criteria for the AS protocol. The stringent follow-up of PCa patients under AS is necessary to implement the necessary treatment as soon as there is disease progression or the cancer becomes aggressive.

\section{Acknowledgments}

Jędrzej Dragan and Jagoda Kania have equally contributed to this manuscript and should be considered as first authors.

\section{Conflict of interest}

The authors declare no conflict of interest.

\section{References}

1. Haas GP, Delongchamps N, Brawley OW, Wang CY, de la Roza $G$. The worldwide epidemiology of prostate cancer: perspectives from autopsy studies. Can J Urol 2008; 15: 3866-71.

2. Golabek T, Bukowczan J, Sobczynski R, Leszczyszyn J, Chlosta PL. The role of micronutrients in the risk of urinary tract cancer. Arch Med Sci 2016; 12: 436-47.

3. Zlotta AR, Egawa S, Pushkar D, et al. Prevalence of prostate cancer on autopsy: cross-sectional study on unscreened Caucasian and Asian men. J Natl Cancer Inst 2013; 105: 1050-8.
4. National Institute for Health and Care Excellence. Prostate cancer: diagnosis and management. NICE 2014.

5. Alam R, Carter HB, Epstein JI, Tosoian JJ. Active surveillance of prostate cancer: current state of practice and utility of multiparametric magnetic resonance imaging. Rev Urol 2017; 19: 77-88.

6. Van Den Bergh RC, Ahmed HU, Bangma CH, Cooperberg MR, Villers A, Parker CC. Novel tools to improve patient selection and monitoring on active surveillance for low-risk prostate cancer: a systematic review. Eur Urol 2014; 65: 1023-31.

7. Morash CT, Tey R, Agbassi C, et al. A Quality Initiative of the Program in Evidence-Based Care (PEBC), Cancer Care Ontario (CCO): Active Surveillance for the Management of Localized Prostate Cancer. Cancer Care Ontario 2014; Evidence-Based Series 17-9.

8. Herden J, Weissbach L. Utilization of Active Surveillance and Watchful Waiting for localized prostate cancer in the daily practice. World J Urol 2018; 36: 383-91.

9. Glaser ZA, Gordetsky JB, Porter KK, Varambally S, Rais-Bahrami S. Prostate cancer imaging and biomarkers guiding safe selection of active surveillance. Front Oncol 2017; 7: 256.

10. Clinical NCCN Guidelines P Prostate Cancer Early Detection. 2015;

11. Mottet N, Bellmunt J, Briers E, et al. EAU guidelines on prostate cancer. Actas Urol European Association of Urology 2015.

12. Hamdy FC, Donovan JL, Lane JA, et al. 10-Year outcomes after monitoring, surgery, or radiotherapy for localized prostate cancer. N Engl J Med 2016; 375: 1415-24.

13. Czerw Al, Religioni U, Deptala A, Fronczak A. Pain, acceptance of illness, adjustment to life with cancer and coping strategies in prostate cancer patients. Arch Med Sci 2017; 13: 1459-66.

14. Butow P, Bell M, Smith A, Fardell J. 2013 Conquer fear: protocol of a randomised trial od a psychological intervention to reduce fear of cancer recurrence. BMC Cancer 2013; 13: 201.

15. Chung MS, Lee SH. Current status of active surveillance in prostate cancer. Investig Clin Urol 2016; 57: 14.

16. Bokhorst LP, Valdagni R, Rannikko A. A decade of active surveillance in the PRIAS study: an update and evaluation of the criteria used to recommend a switch to active treatment. Eur Urol 2016; 70: 954-60.

17. Welty CJ, Cowan JE, Nguyen H. Extended follow-up and risk factors for disease reclassification in a large active surveillance cohort for localized prostate cancer. J Urol 2015; 193: 807-11.

18. Selvadurai ED, Singhera M, Thomas K. Medium-term outcomes of active surveillance for localised prostate cancer. Eur Urol 2013; 64: 981-7.

19. Thompson JE, Hayen A, Landau A, et al. Medium-term oncological outcomes for extended vs saturation biopsy and transrectal vs transperineal biopsy in active surveillance for prostate cancer. BJU Int 2015; 115: 884-91.

20. Thomsen FB, Berg KD, Roder MA. Active surveillance for localized prostate cancer: an analysis of patient contacts and utilization of healthcare resources. Scand J Urol 2015; 49: 43-50.

21. Soloway MS, Soloway CT, Eldefrawy A. Careful selection and close monitoring of low-risk prostate cancer patients on active surveillance minimizes the need for treatment. Eur Urol 2010; 58: 831-5.

22. Klotz L, Vesprini D, Sethukavalan P. Long-term follow-up of a large active surveillance cohort of patients with prostate cancer. J Clin Oncol 2015; 33: 272-7. 
23. Tosoian JJ, Mamawala M, Epstein JI. Intermediate and longer-term outcomes from a prospective active-surveillance program for favorable-risk prostate cancer. J Clin Oncol 2015; 33: 3379-85.

24. PIRADS Prostate Imaging-Reporting Data System v2 2015. American College of Radiology 2015.

25. Hoffmann R, Logan C, O'Callaghan M, Gormly K, Chan K, Foreman D. Does the Prostate Imaging-Reporting and Data System (PI-RADS) version 2 improve accuracy in reporting anterior lesions on multiparametric magnetic resonance imaging (mpMRI)? Int Urol Nephrol 2018; 50: 13-9.

26. Kasel-Seibert M, Lehmann T, Aschenbach R, et al. Assessment of PI-RADS V2 for the detection of prostate cancer. Eur J Radiol 2016; 85: 726-31.

27. Barentsz JO, Weinreb JC, Verma S, Thoeny HC, Tempany CM, Shtern F. Synopsis of the PI-RADS v2 guidelines for multiparametric prostate magnetic resonance imaging and recommendations for use. Eur Urol 2016; 69: 41-9.

28. Scialpi M, D'Andrea A, Martorana E, et al. Biparametric MRI of the prostate. Turk J Urol 2017; 43: 401-9.

29. Lai WS, Gordetsky JB, Thomas JV, Nix J, Rais-Bahrami S. Factors predicting prostate cancer upgrading on magnetic resonance imaging-targeted biopsy in an active surveillance population. Cancer 2017; 123: 1941-8.

30. Pepe P, Cimino S, Garufi A, et al. Confirmatory biopsy of men under active surveillance: extended versus saturation versus multiparametric magnetic resonance imaging/transrectal ultrasound fusion prostate biopsy. Scand J Urol 2017; 51: 260-3.

31. Pepe P, Cimino S, Garufi A, Priolo G, Russo GI, Giardina R. Detection rate for significant cancer at confirmatory biopsy in men enrolled in active surveillance protocol: 20 cores vs 30 cores vs MRI/TRUS fusion prostate biopsy. Arch Ital Urol Androl 2016; 88: 300-3.

32. Bancroft EK, Page EC, Castro E, et al. Targeted prostate cancer screening in BRCA1 and BRCA2 mutation carriers: results from the initial screening round of the IMPACT study. Eur Urol 2014; 66: 489-99.

33. Pezaro C, Woo HH, Davis ID. Prostate cancer: measuring PSA. Intern Med J 2014; 44: 433-40.

34. Fradet V, Toren P, Nguile-Makao M, Lodde M. Prognostic value of urinary prostate cancer antigen 3 (PCA3) during active surveillance of patients with low-risk prostate cancer receiving5a-reductase inhibitors. BJU Int 2018; 121: 399-404.

35. Bernardeau S, Charles T, Fromont-Hankard G, Irani J. The role of a single PCA3 test before a first negative prostate biopsy: 5-year follow-up. Prog Urol 2017; 27: 325-30.

36. Sanda MG, Feng Z, Howard DH, et al. Association between combined TMPRSS2:ERG and PCA3 RNA urinary testing and detection of aggressive prostate cancer. JAMA Oncol 2017; 3: 1085-93.

37. Nickens KP, Ali A, Scoggin T, et al. Prostate cancer marker panel with single cell sensitivity in urine. Prostate 2015; 75: 969-75.

38. Kretschmer A, Tilki D. Biomarkers in prostate cancer current clinical utility and future perspectives. Crit Rev Oncol Hematol 2017; 120: 180-93.

39. Tosoian JJ, Druskin SC, Andreas D, et al. Prostate Health Index density improves detection of clinically significant prostate cancer. BJU Int 2017; 120: 793-8.

40. Index PH. The Prostate Health Index (phi) Superior Performance Demonstrated through Multiple The Prostate Health Index: 2015.

41. Van Neste L, Hendriks RJ, Dijkstra S, et al. Detection of high-grade prostate cancer using a urinary molecular biomarker-based risk score. Eur Urol 2016; 70: 740-8.
42. Stewart GD, Van Neste L, Delvenne P. Clinical utility of an epigenetic assay to detect occult prostate cancer in histopathologically negative biopsies: results of the MATLOC study. J Urol 2013; 189: 1110-6.

43. Navaei AH, Walter BA, Moreno V, Pack SD, Pinto P, Merino $M J$. Correlation between ERG fusion protein and androgen receptor expression by immunohistochemistry in prostate, possible role in diagnosis and therapy. J Cancer 2017; 8: 2604-13

44. Yu J, Yu J, Mani RS, et al. An integrated network of androgen receptor, polycomb, and TMPRSS2-ERG gene fusions in prostate cancer progression. Cancer Cell 2010; 17: 443-54. 